Article

\title{
Ultra-High Molecular Weight Polyethylene Reinforced with Multiwall Carbon Nanotubes: In Vitro Biocompatibility Study Using Macrophage-Like Cells
}

\author{
Nayeli Camacho ${ }^{1, *}$, Stephen W. Stafford ${ }^{1}$, Kristine M. Garza ${ }^{2}$, Raquel Suro ${ }^{3}$ \\ and Kristina I. Barron ${ }^{3}$
}

1 Metallurgical and Materials Engineering Department, University of Texas at El Paso, El Paso, TX 79968, USA; E-Mail: stafford@utep.edu

2 Border Biomedical Research Center, University of Texas at El Paso, El Paso, TX 79968, USA; E-Mail:kgarza@utep.edu

3 Biological Science Department, University of Texas at El Paso, El Paso, TX 79968, USA; E-Mails: raquelmsuro@gmail.com (R.S.); kibarron@miners.utep.edu (K.I.B.)

* Author to whom correspondence should be addressed; E-Mail: ncamacho@miners.utep.edu; Tel.: +1-915-747-6930; Fax: +1-915-747-8036.

Academic Editor: James E. Krzanowski

Received: 1 June 2015 / Accepted: 22 July 2015 / Published: 31 July 2015

\begin{abstract}
Carbon nanotubes are highly versatile materials; new applications using them are continuously being developed. Special attention is being dedicated to the possible use of multiwall carbon nanotubes in biomaterials contacting with bone. This study describes the response of murine macrophage-like Raw 264.7 cells after two and six days of culture in contact with artificially generated particles from both, ultra-high molecular weight polyethylene polymer and the composite (multiwall carbon nanotubes and ultra-high molecular weight polyethylene). This novel composite has superior wear behavior, having thus the potential to reduce the number of revision knee arthroplasty surgeries required by wear failure of tibial articulating component and diminish particle-induced osteolysis. The results of an in vitro study of viability, and interleukin-6 and tumor necrosis factor-alpha production suggest good cytocompatibility, similar to that of conventional ultra-high molecular weight polyethylene.
\end{abstract}

Keywords: in vitro macrophages response; knee replacement; multiwall carbon nanotubes; ultra-high molecular weight polyethylene; wear debris cytotoxicity 


\section{Introduction}

Knees carry half of the body weight and provide support and mobility to the human body. In recent years, the incidence of joint degeneration has increased considerably in the young and elderly population; the National Center for Health Science reported that there are more than 300,000 knee replacements per year in the United States [1]. In total knee replacements (TKRs) the production of wear debris is expected because of the sliding and rotating movements of the femoral component against the bearing surface. The articular component material must be completely biocompatible to avoid strong immune reactions due to the interaction of this wear debris with the human body fluids and tissues surrounding the knee joint. Wear debris and degradation products created while using the implant should not cause any inflammatory responses or secondary effects.

Ultra-high molecular weight polyethylene (UHMWPE) has been proven to be a good counterpart material when articulating against cobalt-chromium-molybdenum (Co-Cr-Mo) femoral components in TKRs $[2,3]$. It displays a very low friction coefficient and it is widely used in the orthopaedic field as a bearing surface in different artificial joints. Because of its high wear resistance and high impact strength, this material remains the material of choice for the fabrication of articular tibial components. Nevertheless, its performance is affected by high creep when compared to metal and bone [4,5]. Also, there has been ongoing concern about the use of UHMWPE because wear of this material often leads to the generation of numerous micron and submicron sized particles (wear debris), which can cause a number of immunological responses and ultimately lead to osteolysis and loosening of the knee implant [4-6].

One of the most researched areas in the last years is the addition of multiwall carbon nanotubes (MWCNT for different applications in the biomedical field; nonetheless, before such material can be incorporated into biomedical devices, the toxicity and biocompatibility of CNTs needs to be thoroughly investigated [7]. The effect of carbon nanotubes on living organisms has been recently studied [7,8]. However, the results of these studies remain controversial [2,3]. Even though carbon nanotubes seem to be a viable option to improve the tribological properties of polymer/composite materials used in orthopedic implants, the biological response of UHMWPE reinforced with carbon nanotubes remains unknown [2,3,9]. This study will focus on the analysis of UHMWPE reinforced with multiwall carbon nanotubes (UHMWPE/CNT) and its cytotoxicity when compared to commercial UHMWPE. The usefulness of UHMWPE/CNT composite must be accompanied by a positive cell interaction to be considered as a viable biomaterial for orthopedic implant consideration.

\section{Materials and Methods}

The material of interest in this study is the wear debris obtained mechanically from produced samples made from a composite formed by a polymer matrix (UHMWPE) and MWCNT, which is simultaneously compared to the wear debris of samples produced using UHMWPE (orthopedic grade). The multiwall carbon nanotubes are commercial products that were purchased from Sky Spring Nanomaterials, USA. According to the specifications of the manufacturer, the nanotubes are $20-30 \mathrm{~nm}$ 
in diameter and about $10-30 \mu \mathrm{m}$ in length. Their characteristics, as specified by the manufacturer, are display in Table 1. In literature, one aspect that is considered for MWCNTs to be used in biomedical applications is the purity of this material; this is essential to avoid detrimental reactions when the material is in contact with the human body [8]. Moreover, the XEDS spectrum of the MWCNTs was obtained in a Hitachi S4800 field emission scanning electron microscope (FESEM) and is displayed on Figure 1.

Table 1. Multiwall nanotubes (MWCNTs) general characteristics as specified by the manufacturer.

\begin{tabular}{cc}
\hline Multiwall Nanotubes (MWCNTs) & Characteristics \\
\hline Purity & $>95 \mathrm{wt} \%$ \\
Outside diameter & $20-30 \mathrm{~nm}$ \\
Inside diameter & $5-10 \mathrm{~nm}$ \\
Length & $10-30 \mu \mathrm{m}$ \\
SSA & $>110 \mathrm{~m}^{2} / \mathrm{g}$ \\
Ash & $<1.5 \mathrm{wt} \%$ \\
Amorphous carbon & $<3.0 \%$ \\
Electrical conductivity & $>100 \mathrm{~s} / \mathrm{cm}^{3}$ \\
Bulk density & $0.28 \mathrm{~g} / \mathrm{cm}^{3}$ \\
True density & $\sim 2.1 \mathrm{~g} / \mathrm{cm}^{3}$ \\
Manufacturing method & Catalytic CVD \\
\hline
\end{tabular}

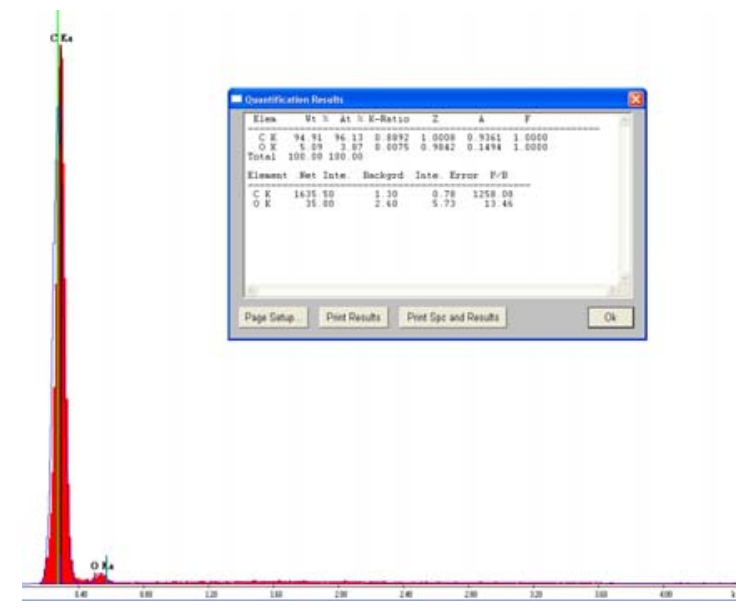

Figure 1. XEDS analysis displaying chemical composition of carbon nanotubes in as-received conditions.

The XEDS analysis did not revealed the presence of any impurities other than oxygen in the carbon nanotubes. Additionally, UHMWPE powder, engineered CNTs and the composite, in a powder form, were tested to observe how the cells reacted to each individual material and the effect of the material size. The materials were tested with the murine macrophage cell line Raw264.7. Macrophages (MФ) are part of the innate immune system, and their main function is to engulf and digest any cellular debris or pathogen present in the body. The focus of the study was to examine the cell response in the presence of UHMWPE/MWCNTs and determine the cytokine production, specifically, the production of Interleukin-6 (IL-6) and tumor necrosis factor-alpha (TNF- $\alpha$ ), pro-inflammatory markers. 


\subsection{Nanocomposite Material}

Medical grade UHMWPE was supplied by Ticona Engineering Polymers, Inc. (Bishop, TX, USA); the trade name of the material used in this study was GUR ${ }^{\circledR} 1020$. The manufacturer specifications of UHMWPE powder were: density $=0.930 \mathrm{~g} / \mathrm{m}^{3}$, mean particle size $137 \mu \mathrm{m}$, and average molecular weight $=3.5$ million $\mathrm{g} / \mathrm{mol}$. The MWCNTs were purchased from Sky Spring Nanomaterials (Houston, TX, USA). The manufacturer specifications of CNTs were: diameter range $=20-30 \mathrm{~nm}$, length of the tubes $=10-30 \mu \mathrm{m}$, and purity $>95 \%$.

The CNTs were mixed with UHMWPE using the experimental protocol developed Xue et al. [10] to produce a homogeneous mixture to be used as the raw material in a compression molding machine. Figure 2 presents a SEM image of the raw composite material, where it can be noticed that the nanotubes are entangled on the UHMWPE particles. The compressed samples of both UHMWPE polymer and UHMWPE/CNT nanocomposites (1.25 wt $\%$ of MWCNT) were prepared under optimized testing conditions of pressure, temperature, and time, as determined from our previous studies [10,11].

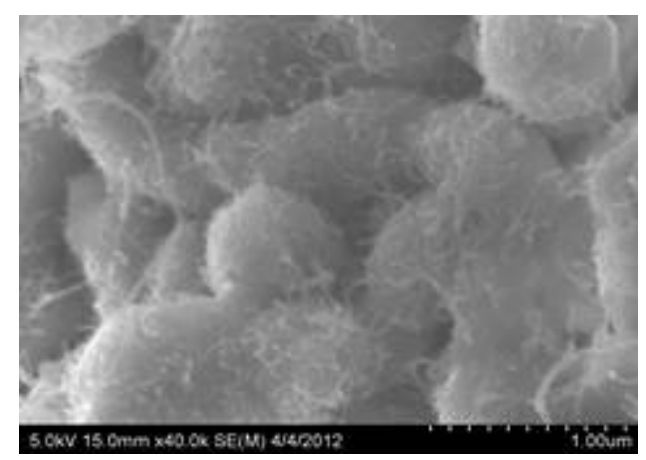

Figure 2. Micrographs of composite powder at $40 \mathrm{kx}$.

Hardness is defined as the resistance of a material to penetration; in the case of polymers, hardness is usually measured with a durometer that measures the resistance of the plastic to penetration of a spring loaded needle-like indenter by Shore D scale, in the case hard "plastics". In order to assess the effect of carbon nanotubes on the hardness of UHMWPE, twelve different concentrations were tested, ranging from 0.25 to $20 \mathrm{wt} \%$. From the hardness results, an optimal concentration was chosen for the cytotoxicity studies.

The wear particles from the polymer and the nanocomposite were generated mechanically in the presence of ethanol. After draining the ethanol from the system, the particles were collected and oven-dried, and then sterilized by $\gamma$ rays at a dose of $25 \mathrm{kGy}$ in a X-Rad 160 irradiatiot. The sterilized particles were used as the raw material in this study. Micrographs of the generated wear particles show that the particles of both materials are in the form of fibrils and have an irregular shape. The size of the particles $(0.5-2 \mu \mathrm{m})$ was comparable to the phagocyte range for biological response studies associated with secretion of cytokines inductive of osteolysis, as described by Green et al. [12], Revell [5], Kurtz [2] and Mcgee [6]. 


\subsection{Dose Response}

The control group comprised Raw264.7 macrophage-like cells that were seeded in the wells of 12 -well plates at a density of 100,000 cells/well and cultured in the presence of growth medium. Cells were grown in HyClone DMEM hyglucose culture media, 10\% fetal bovine serum (FBS), 1\% 1-glutamin, $1 \%$ of antibiotics (penicillin and streptomycin) and 1\% of sodium bicarbonate per container of $500 \mathrm{~mL}$ of media. The sterilized particles used in this study (UHMWPE and UHMWPE/CNT particles with an average particle size of $130 \mu \mathrm{m}$, engineered MWNTs and, UHMWPE and UHMWPE/CNT debris) were suspended in dimethylsulfoxyde (DMSO) at a concentration of $5 \mathrm{mg} / \mathrm{mL}$, and vortex to ensure a homogenous suspension of the particles immediately prior to pipetting. The concentrations studied ranged from $1 \mu \mathrm{g} / \mathrm{mL}$ to $50 \mu \mathrm{g} / \mathrm{mL}$ for all treatments. Media and campthotecin were used as positive and negative controls and were included in each experiment; also, DMSO was assessed in the dose response analysis, since DMSO was used as the vehicle control. The particles were added to the cells after the 24-h growth period (day 1); then, the culture plate was placed in the incubator for another $24 \mathrm{~h}$. The medium and treatments were refreshed on day 2, and the cell viability was assessed on day 3. All experiments were carried out in triplicates.

Viability was assessed using a luminescent cell viability assay (Cell Titer-Glo Luminescent). The reagent lyses the cells and interacts with the adenosine triphosphate (ATP) within the cells. When the reagent interacts with the ATP, the cells luminesce and produce a signal that is later on detected by the luminometer, where the viability is assessed; the more luminescence, the higher the viability. Briefly, the viability assessment takes place on day 3 , the cells are scrapped from the well walls and $100 \mu \mathrm{L}$ of the cell suspension from each well are transferred to an opaque $96-w e l l$ plate. Then, $100 \mu \mathrm{L}$ of cell titer Glo Luminescent are added to each well for the viability assay. Medium was added to three wells in the opaque 96-well plate and were used as blanks. The cells well then placed in the luminometer (Luminoskan Ascent from Labsystems), and the viability was assessed. The voltage used was $900 \mathrm{~V}$ and each well was analyzed for $10 \mathrm{~s}$. Once the viability was assessed, the half maximal effective concentration $\left(\mathrm{EC}_{50}\right)$ was established and used in the acute and chronic exposure studies.

\subsection{Acute and Chronic Exposure}

In the acute and chronic exposure, viability and pro-inflammatory cytokine production were assessed to establish the macrophage response to the nano-particles (wear debris and carbon nanotubes) and micro-particles (raw material). Lipopolysaccharide (LPS) endotoxin served as a positive control for the activation of the cells, LPS provides the most robust stimulus to macrophages and it attest to the cell viability and reaction in the culture. All assays were run in duplicates.

Viability for the acute exposure was assessed using the same luminescent cell viability assay as in the dose response and the same protocol was followed: the cells were seeded in 12-well plates at a density of 300,000 cells per well on day 0, the particles were added on day 1 and refreshed on day 2 at a concentration of $12.5 \mu \mathrm{g} / \mathrm{mL}$, the LPS and negative control were also activated on day 3 , and the viability was assessed in the luminometer on day 3. On the other hand, for the chronic exposure, cells were seeded on day 0 in 12-well plates at a density of 40,000 cells per well. The particles were added 
on day 1 and refreshed on days 4 and 6 . The LPS and camptothecin controls were activated on day 6 as well, and finally, viability was assessed in the luminometer on day 7.

Pro-inflammatory cytokine production, specifically, TNF- $\alpha$ and IL-6, were assessed using commercially available enzyme-linked immunosorbent assays (ELISA) kits (BioLegend and BD Biosciences, San Diego, CA, USA). The ELISA analyses were performed according to manufacturer's instructions. For the TNF- $\alpha$ production, microtiter ELISA plates were coated with anti-TNF $\alpha$ capture antibody over night at $4{ }^{\circ} \mathrm{C}$. The ELISA kit used to measure the IL-6 production included coated plates with anti-IL-6 capture antibody. The plates were then blocked at room temperature with 3\% bovine serum albumin in deionized water. Cell culture supernatants from treated $M \Phi$ were added to the plates. Following binding of cytokines to the capture antibodies, the plates were incubated with biotinconjugated anti-TNF $\alpha$ and IL-6 antibodies, respectively, followed by streptavidin-HRP (Biosource/Life Technologies, Grand Island, NY, USA). The chromogenic substrate 3,3'5,5'-tetramethylbenzidine (TMB) (BD OptEIA, San Jose, CA, USA) was utilized for color development and absorbance was measured by microplate spectrophotometer (VersaMax Microplate Reader, Molecular Devices, Silicon Valley, CA, USA). Cytokine concentrations were calculated against murine recombinant cytokines (BD Pharmingen, San Jose, CA, USA).

\section{Results and Discussion}

\subsection{Hardness Testing}

Hardness of pure and reinforced samples was measured with a durometer. In order to understand the effect of carbon nanotubes in the polymer matrix, samples with twelve different concentrations were also fabricated (See Table 2). The load applied on each sample, according to the specifications of the Shore D scale for UHMWPE (ASTM standard D2240), was $4536 \mathrm{~g}$. Figure 3 displays the average hardness value dependig on CNTs concentration. From the graph in Figure 3, it can be concluded that low and high concentrations of CNTs are detrimental for UHMWPE, since these concentrations present the lowest hardness values.

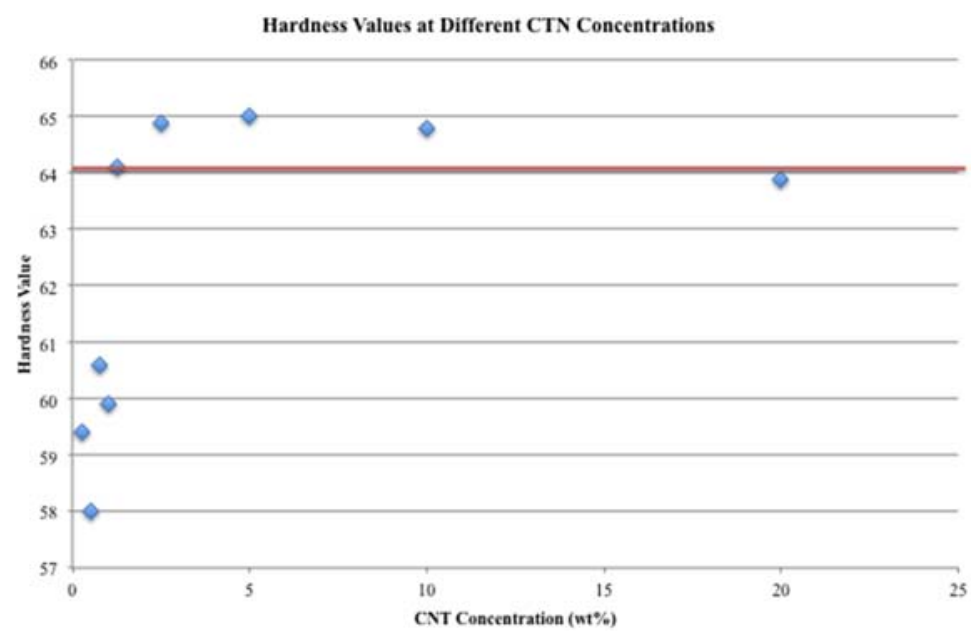

Figure 3. Graph displaying CNT effect on hardness values. The red line stands for the hardness value of the CNT concentration used in this study. 
Table 2. Experimental hardness of nanocomposite at different CNT concentrations.

\begin{tabular}{cccccccccc}
\hline Machine & \multicolumn{1}{l}{ Asker Durometer Model EX-D Serial No. 004011 Type D } \\
\hline Scale & \multicolumn{1}{l}{ Shore D } \\
\hline Analysis & \multicolumn{1}{l}{ CNT Concentration Effect on Hardness } \\
\hline Test date & 11-Jun-12 \\
\hline CNT & \multicolumn{1}{l}{ Indent } \\
\hline Percentage & $\mathbf{1}$ & $\mathbf{2}$ & $\mathbf{3}$ & $\mathbf{4}$ & $\mathbf{5}$ & $\mathbf{6}$ & $\mathbf{7}$ & $\mathbf{8}$ & Average \\
\hline 0 & 60 & 60 & 62 & 61 & 62 & 62 & 62 & 61 & 61.5 \\
0.25 & 59 & 61 & 60 & 58 & 58 & 59 & 60 & 60 & 59.4 \\
0.50 & 57 & 57 & 58 & 58 & 58 & 59 & 58 & 59 & 58.0 \\
0.75 & 60 & 59 & 61 & 61 & 61 & 61 & 61 & 61 & 60.6 \\
1.00 & 59 & 60 & 60 & 61 & 60 & 60 & 60 & 59 & 59.9 \\
1.25 & 64 & 64 & 64 & 64 & 64 & 65 & 64 & 64 & 64.1 \\
2.50 & 65 & 64 & 65 & 65 & 66 & 64 & 65 & 65 & 64.9 \\
5.00 & 65 & 64 & 64 & 66 & 66 & 65 & 64 & 66 & 65.0 \\
10.00 & 65 & 64 & 64 & 64 & 66 & 64 & 66 & 65 & 64.8 \\
20.00 & 63 & 64 & 64 & 64 & 64 & 65 & 64 & 63 & 63.9 \\
\hline
\end{tabular}

The wear particles from the polymer and the nanocomposite generated mechanically were observed under the scanning electron microscope at $15 \mathrm{kV}$ (SEM-Hitachi TM-1000) and they are displayed in Figure 4. The polymer and nanocomposite particles displayed irregular shapes and variable size. However, the nanocomposite debris was characterized by its fibrillar structure.
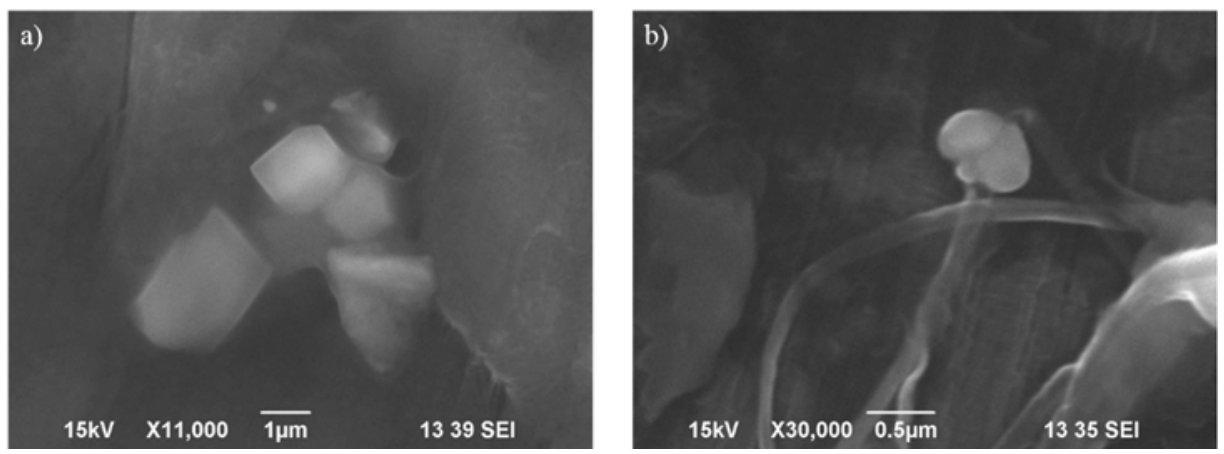

Figure 4. Polymer (a) and nanocomposite (b) wear debris micrographs.

\subsection{Dose Response}

The results from the dose response and viability assays showed that UHMWPE micro-particles displayed the lowest $\mathrm{EC}_{50}$ (between 6-7 $\mu \mathrm{g} / \mathrm{mL}$ ) while the UHMWPE wear debris displayed an $\mathrm{EC}_{50}$ between $10-15 \mu \mathrm{g} / \mathrm{mL}$, both of these are way below the $\mathrm{EC}_{50}$ from the DMSO, which is the vehicle control. In other words, from the dose response it can be concluded that UHMWPE is cytotoxic in a micro and nano-scale. On the other hand, UHMWPE/CNT in any of its forms did not reach the EC50; because of this, additional concentrations had to be explored. For the composite treatments, 5 additional concentrations were analyzed from 50 to $100 \mu \mathrm{g} / \mathrm{mL}$. Nevertheless, the EC50 for UHMWPE/CNT debris was about $66 \mu \mathrm{g} / \mathrm{mL}$ while the EC50 for the UHMWPE/CNT micro-particles could not be reached. 


\subsection{Acute and Chronic Exposure}

In the acute and chronic exposure it was necessary to use a single dose for all the treatments. Moreover, because this is a comparative study between the cytotoxicity of UHMWPE and the UHMWPE/CNT, it was important to assess the cells under the same conditions. It was decided that the dose at which the $\mathrm{EC}_{50}$ for the UHMWPE debris was reached, was the most adequate for the assays. Figures 5 and 6 display the results of the viability assays performed for the acute and chronic exposure, respectively.

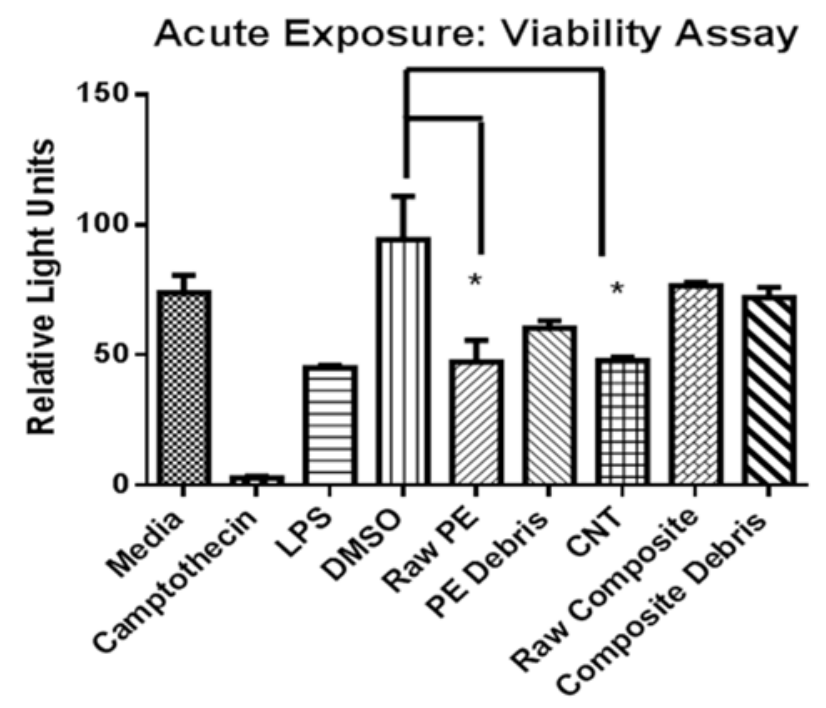

Figure 5. Viability assay for the acute exposure.

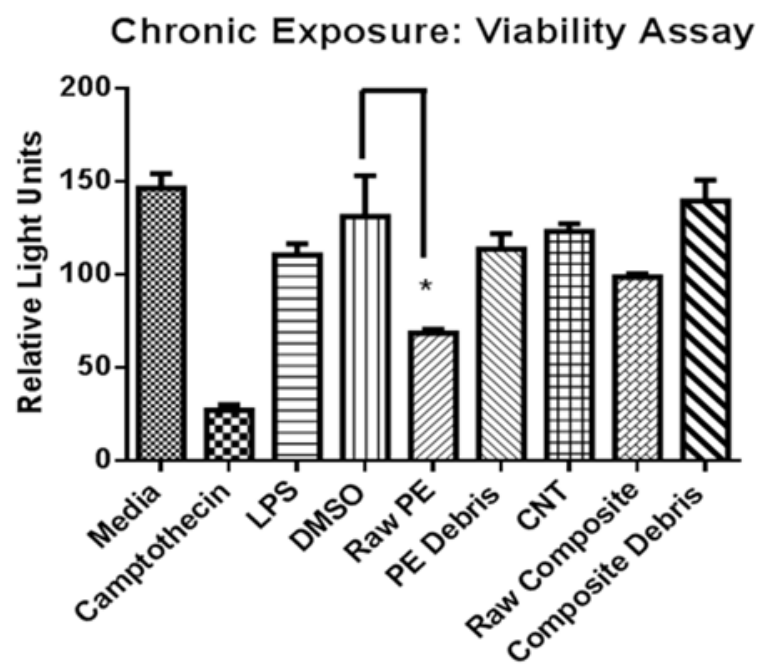

Figure 6. Viability assay for the chronic exposure.

The viability and proliferation assays (acute and chronic exposure) showed no significant differences between the control (DMSO) and UHMWPE/CNT in any of its forms. As a matter of fact, in the chronic exposure, UHMWPE/CNT displayed a viability of $98.5 \% \pm 4.6 \%$ (raw material) and $75.3 \% \pm 1.1 \%$ (wear debris) while UHMWPE displayed a viability of $86.6 \% \pm 3.4 \%$ and $52.2 \% \pm$ $1.2 \%$ for the raw material and the debris, respectively. Comparing viability results in the chronic and 
acute exposure, it can be concluded that $\mathrm{M} \Phi$ recover from the initial exposure to UHMWPE in its raw form, since viability when from $50.1 \% \pm 4.5 \%$ to $86.6 \% \pm 3.4 \%$. On the other hand, the opposite effect was seen in the UHMWPE wear debris, with this material, the longer the exposure, the lower viability values are seen. The same effect is observed in the presence of UHMWPE/CNT. Nevertheless, the decrement in vitality is less obvious.

Tumor necrosis factor alpha (TNF- $\alpha$ ) production for acute and chronic exposure where measured by ELISA and the results are displayed in Figures 7 and 8. TNF- $\alpha$ production in the presence of the particles was compared to the negative control (media). Furthermore, the results of UHMWPE where compared to those obtained for the polymer/composite. All tests were run three times and in duplicates. From the graph in Figure 7 it can be observed that there is a significant difference (39.8\%) in the TNF- $\alpha$ production when the cells are exposed to UHMWPE alone and when they are exposed to the reinforced polymer at $48 \mathrm{~h}$. However, when the exposure time increases, the difference between the TNF- $\alpha$ production becomes non-significant. Another conclusion that can be drawn from this analysis is that bigger particles will increase the inflammatory response of the cells.

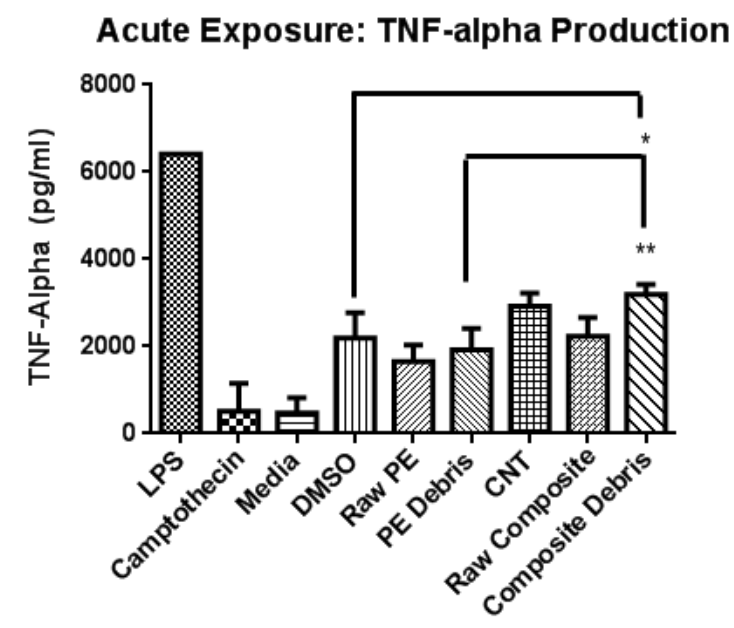

Figure 7. TNF- $\alpha$ production after $48 \mathrm{~h}$ of exposure.

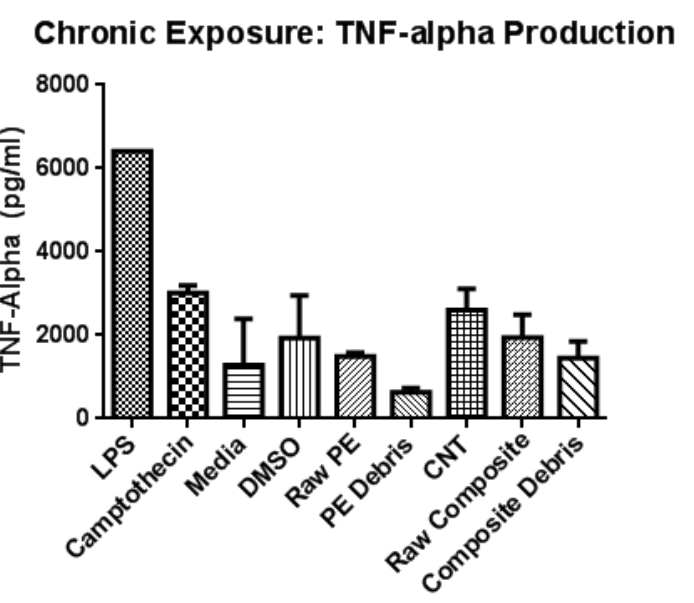

Figure 8. TNF- $\alpha$ production after $168 \mathrm{~h}$ of exposure. 
Finally, IL-6 production was also assessed to determine the inflammatory response to the cells in the presence of the different particles. Figures 9 and 10 display the graphs with the results obtained in the acute and chronic exposure. Even though UHMWPE stimulated the cells enough for them to produce IL-6, the amount that the MФ produced is extremely low. Nevertheless, it has to be mentioned that UHMWPE/CNT did not cause any effect on the cells when it referred to the IL-6 production. These results are in agreement with those reported by Chlopek et al. [13] where IL-6 production was measured in the presence of polysulfone (PSU) with high purity carbon nanotubes. Additionally, this study measured the potential of macrophages to release free radicals when exposed to carbon nanotubes or carbon particles of similar dimensions [13]. Chlopek et al. [13] confirmed a good biocompatibility of carbon nanotubes, characterized by a lack of IL-6 production as well as free radicals induction.

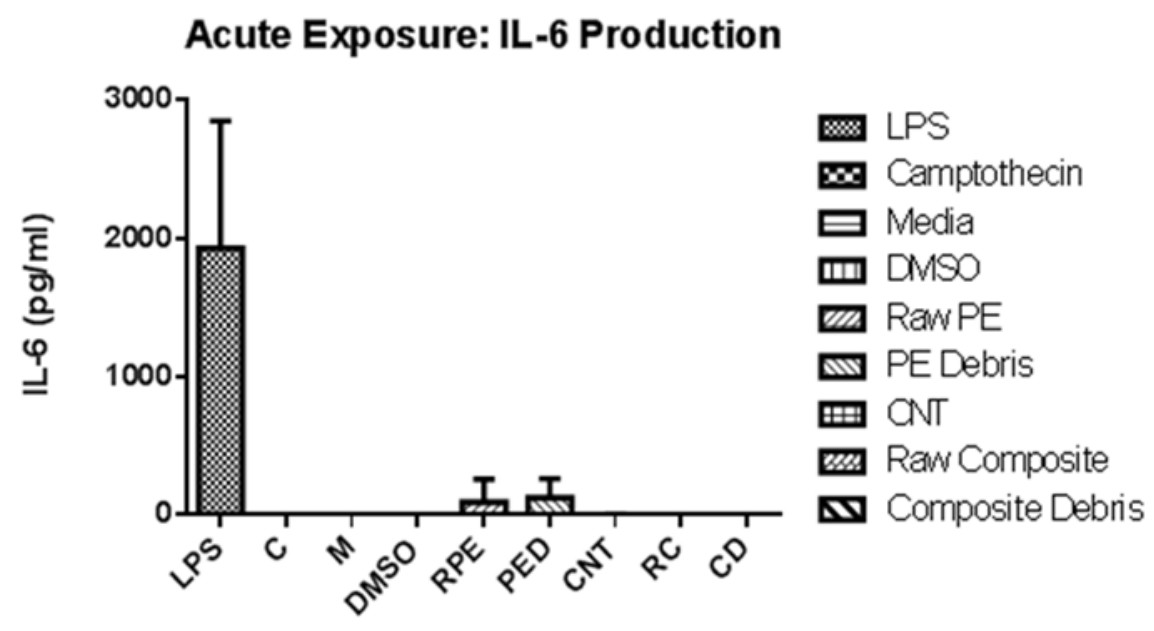

Figure 9. IL-6 production after $48 \mathrm{~h}$ of exposure.

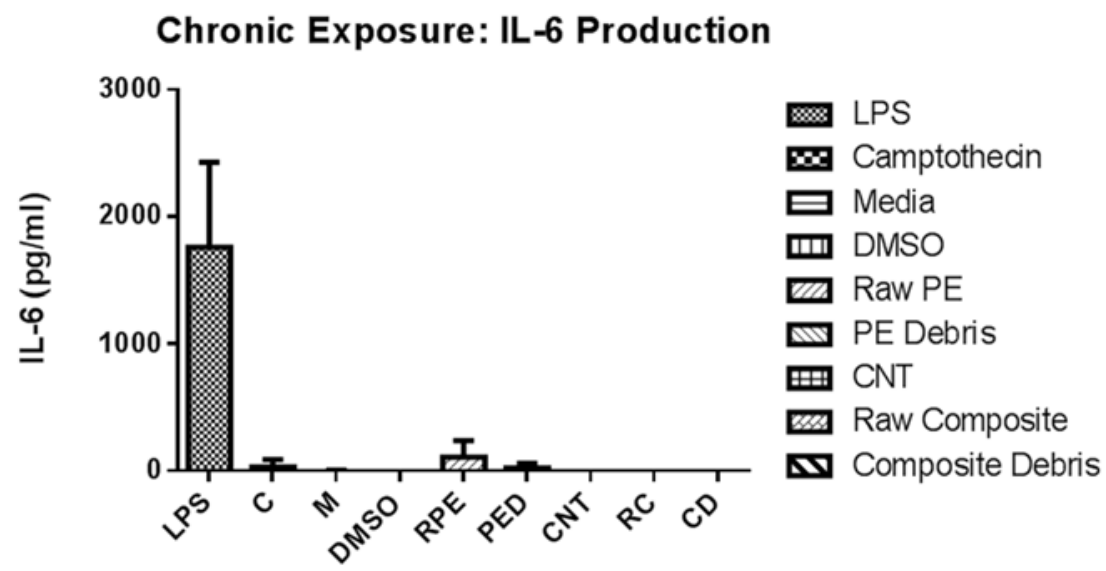

Figure 10. IL-6 production after $168 \mathrm{~h}$ of exposure.

Figure 11 displays cells that have been exposed to different particles. Photographs A and B display M $\Phi$ exposed to UHMWPE, in its raw and debris form, respectively, while photographs C and D display MФ exposed to UHMWPE/CNT, also in its raw and debris form, respectively. As it can be observed from the photograph $\mathrm{B}$, the cells seemed to have morphed their appearance more and their pseudopods are larger when compared to those on the other images. Moreover, the cells appeared to be 
less confluent in presence of UHMWPE debris. For cells exposed to the polymer/composite, the micro size composite seemed to have enlarged the cells when compared to the debris. Nonetheless, the shape of the cells and confluence in the wells appears to be similar.

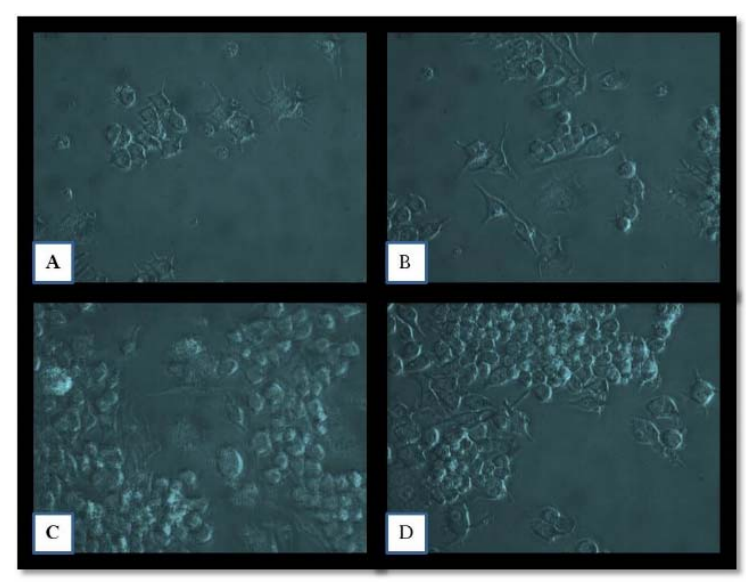

Figure 11. Micrographs of cells after $48 \mathrm{~h}$ of exposure to different micro and nano particles (160X). (A) Raw UHMPWE, (B) UHMWPE debris, (C) Raw UHMWPE/CNT, and (D) UHMWPE/CNT debris.

\subsection{Discussion}

Different concentrations of carbon nanotubes in the polymeric matrix were evaluated for this study. Hardness testing showed higher values for UHMWPE/CNT at concentrations above $1 \mathrm{wt} \%$ and below $42 \mathrm{wt} \%$ CNT than that obtained for pure UHMWPE. The increase in hardness was not very significant from the chosen CNT concentration (1.25 wt\%) (2.6 points in the D-Shore scale). However, a small increment in hardness value translates into better creep and wear resistance. It was concluded that the higher the concentration of carbon nanotubes, the higher the values for hardness testing. However, it was noted that after an addition of about $10 \mathrm{wt} \%$ or more, the hardness values plateaued. Also it was noted that when the addition of carbon nanotubes exceeded $20 \mathrm{wt} \%$, lower hardness values were obtained.

Viability, and tumor necrosis factor alpha (TNF- $\alpha$ ) and interleukin-6 (IL-6) production were assessed by macrophage Raw264.7 cells. The results of this in vitro study suggest good cytocompatibility of the UHMWPE/CNT material when compared to UHMWPE (current commercial material); these results are in agreement with those reported by Reis et al. [8] in his studies with osteoblasts. Viability assays demonstrated that in an initial exposure (acute study), macrophages react strongly to particles with the micron and sub-micron size particles (debris). However, when the cells stay in the presence of the material, bigger size particles (raw material) decreases the viability. Even though there were no significant differences between the cells viability when exposed to UHMWPE than when exposed to UHMWPE/CNT, cell viability was slightly higher in the presence of UHMWPE/CNT, especially in its debris form. On the other hand, when pro-inflammatory cytokine production, specifically TNF- $\alpha$ and IL-6, was measured, it was noted that a while IL- 6 was barely present in the supernatants obtained after 48 and $168 \mathrm{~h}$ of exposure to the particles, TNF- $\alpha$ levels were relatively high, especially for the polymer/composite debris after only $48 \mathrm{~h}$ of exposure. Nonetheless, 
TNF- $\alpha$ levels stabilized themselves and no significant difference can be observed among UHMWPE, polymer/composite, and controls.

The use of carbon nanotubes in the medical field has been questioned in the last years because of the controversial results of the biocompatibility of CNTs. However, in this study, the lack of presence of IL-6 in the composite group suggests good cytocompatibility, since IL-6 is a powerful inductor of bone resorption through osteoclast activation. The use of this novel composite in the orthopaedic field could benefit patients in different ways. The composite has demonstrated to be not only superior in mechanical properties, but also it seems that the use of this material could decrease the number of total knee replacement failures due to osteolysis.

\section{Conclusions}

The cellular response associated with engineered wear debris from UHMWPE (current commercial material for tibial articular inserts in TKRs) was compared to the cellular response associated with wear debris from UHMWPE/CNT samples. Murine macrophage-like cells (Raw264.7) were used to assess the cytotoxicity of these materials. A dose response was first performed to establish the $\mathrm{EC}_{50}$ to be used in the acute and chronic exposure to the micro and nanoparticles. Viability was assessed through a luminescent cell viability assay and the cytokines production, specifically TNF- $\alpha$ and IL-6, were assessed by ELISA.

The viability and pro-inflammatory cytokine production was assessed by macrophage-like cells Raw264.7. The main purpose of this study was to compare the cytotoxicity of UHMWPE and UHMWPE/CNT. In the first stage of this research, a dose response assessment was performed to calculate the half maximal effective concentration $\left(\mathrm{EC}_{50}\right)$ to be used in the acute and chronic exposure. The dose response was assessed through the viability of the cells with a commercial luminescent cell viability assay. The results of the dose response display a higher EC50 for UHMWPE/CNT in both forms (raw material and artificially generated debris) than that found for UHMWPE.

The viability assessment in an acute and chronic exposure displayed similar results. There were no significant difference between UHMWPE and the composite or the controls and the composite. However, UHMWPE in its raw form displayed a significant difference when compared to the vehicle control (DMSO). This suggests that the presence of carbon nanotubes in the polymer matrix actually protects the cells from the plastic and increases the viability of $M \Phi$.

On the other hand, pro-inflammatory cytokine production assessment showed different results. UHMWPE/CNT displays a higher production of TNF- $\alpha$ than UHMWPE, especially in micron and sub-micron size (wear debris) in an initial exposure (acute). Nevertheless, the chronic exposure revealed similar levels of TNF- $\alpha$ production in controls, UHMWPE, and polymer/composite. It was noted that in the chronic exposure, bigger particles (raw materials) elicit a higher cytokine production. Finally, the production of IL-6 was assessed by ELISA. From the different materials studied, UHMWPE was the only one that produced IL-6. However, the IL-6 production was so low, that it was not significant when compared to the controls. 


\section{Acknowledgment}

The authors recognize the generous funding contributions of National Council for Science and Technology (CONACyT) to this research effort. This work was also supported by the National Institutes of Health-Research Center at Minority Institutions (5G12RR008124). Kristina Barron was funded by the NIH-Marcus U-Star program (T34-GM008048) and Raquel Suro was funded by the NIH-Rise program for graduate students (R25 GM 069621). The authors would like to thank the UTEP-RCMI Border Biomedical Research Center Core Facilities: Cytometry, Screening, and Imaging; and Biomolecule Analysis.

\section{Author Contributions}

Nayeli Camacho wrote the paper, designed the material and performed its mechanical and biological characterization under the supervision of Stephen W. Stafford and Kristine M. Garza. Additionally, Kristine M. Garza coordinated and supervised the cytotoxicity experiments and contributed to the data analysis. Raquel Suro and Kristina I. Barron contributed to the cytotoxicity experiments and data analysis.

\section{Conflicts of Interest}

The authors declare no conflict of interest.

\section{References}

1. Janeway, P.A. Bioceramics: Materials That Mimic Mother Nature. Am. Ceram. Soc. Bull. 2006, 8, 26-30.

2. Kurtz, S.M. The UHMWPE Handbook: Ultra High Molecular Weight Polyethylene in Total Joint Replacement; Elsevier Inc.: San Diego, CA, USA, 2004.

3. Kurtz, S.M. UHMWPE Biomaterials Handbook, 2nd ed.; Elsevier Inc.: Burlington, MA, USA, 2009.

4. Schmalzried, T.P.; Callaghan, J.J. Current Concepts Review: Wear in Total Knee and Hip Replacements. J. Bone Jt. Surg. 1999, 81, 115-132.

5. Revell, P.A. The Combined Role of Wear Particles, Macrophages and Lymphocytes in the Loosening of Total Joint Replacements. J. R. Soc. Interface 2008, 5, 1263-1278.

6. Mcgee, M.A.; Howie, D.W.; Costi, K.; Haynes, D.R.; Wildenauer, C.I.; Pearcy, M.J.; McLean, J.D. Implant Retrieval Studies of the Wear and Loosening of Prosthetic Joints: Review. Elsevier Sci. Wear 2000, 241, 158-165.

7. Smart, S.K.; Cassady, A.I.; Lu, G.Q.; Martin, D.J. The Biocompatibility of Carbon Nanotubes. Carbon 2006, 44, 1034-1047.

8. Reis, J.; Kanagaraj, S.; Fonseca, A.; Mathew, M.T.; Capela-Silva, F.; Potes, J.; Pereira, A.; Oliveira, M.S.; Simões, J.A. In vitro Studies of Multiwall Carbon Nanotube/Ultrahigh Molecular Weight Polyethylene Nanocomposites with osteoblast-like MG63 Cells. Braz. J. Med. Biol. Res. 2010, 43, 476-482. 
9. Gomez-Barrena, E.; Puertolas, J.A.; Munuera, L.; Konttinen, Y.T. Update on UHMWPE Research: From the Bench to the Bedside. Acta Orthop. 2008, 79, 832-840, doi:10.1080/17453670810016939.

10. Camacho, N. Characterization of Ultra-High Molecular Weight Polyethylene (UHMWPE) Reinforced with Multiwall Carbon Nanotubes for Orthopedic Applications. Ph.D. Dissertation, Materials Science Engineering, University of Texas at El Paso, El Paso, TX, USA, 4 January 2013.

11. Green, T.R.; Fisher, J.; Stone, M.; Wroblewskid, B.M.; Ingham, E. Polyethylene particles of a "critical size" are necessary for the induction of cytokines by macrophages in vitro. Biomaterials 1998, 19, 2297-2302.

12. Yang, X.; Wu, W.; Jacobs, O.; Schädel, B. Tribological behavior of UHMWPE/HDPE blends reinforced with Multi-wall Carbon nanotubes. Polym. Test. 2006, 25, 221-229.

13. Chlopk, J.; Czajkowska, B.; Szaraniec, B.; Frackowiak, E.; Szostak, K.; Béguin, F. In Vitro Studies of Carbon Nanotubes Biocompatibility. Carbon 2006, 44, 1106-1111.

(C) 2015 by the authors; licensee MDPI, Basel, Switzerland. This article is an open access article distributed under the terms and conditions of the Creative Commons Attribution license (http://creativecommons.org/licenses/by/4.0/). 